\title{
Vegetación del Monumento Natural y Paisaje Protegido Loma de Las Tapias. Departamentos Ullum y Albardón, San Juan (ArgentinA)
}

\author{
MARTÍN GUILLERMO ALMIRÓN¹
}

\begin{abstract}
Summary: Vegetation of the Natural Monument and Protected Landscape Loma de Las Tapias. Ullum and Albardón Departaments, San Juan (Argentina). In this study, the vegetation of the Natural Monument and Protected Landscape Loma de Las Tapias (San Juan Province), is described. This protected area was created with the aim to protect the landscape of huayquerías (badlands) and the fossiliferous deposits comprising vertebrate fossils from the Middle Pliocene. To analyze the vegetation, 43 inventories were carried out following the Braun-Blanquet (1979) field methodology, recording environmental variables such as slope, associated geological formation, altitude and vegetal cover. The inventories were grouped through multivariate dendrogrammetric analysis. A total of 72 species of vascular plants was registered. The general physiognomy of the vegetation corresponds to an open xerophilous shrubland dominated by species of Zygophyllaceae and Fabaceae accompanied by other shrubs, cacti and an open herbaceous layer, typical of the Phytogeographic Monte Province. Four vegetation units were detected through the multivariate analysis; these units were related to geology, altitude, slope and plant cover. Since this document could be used by the provincial authorities in charge of the protected area, some topics related to the conservation and use of the area are finally discussed.
\end{abstract}

Key words: Protected areas, Vegetation units, Phytosociology, Hyper arid deserts

Resumen: En el presente trabajo se describe la vegetación del Monumento Natural y Paisaje Protegido Loma de Las Tapias (Provincia de San Juan). Esta área protegida fue creada con el objetivo de custodiar el paisaje de huayquerías y el yacimiento fosilífero de vertebrados del Plioceno Medio. Para analizar la vegetación, se realizaron 43 relevamientos siguiendo la metodología de Braun-Blanquet (1979), registrando variables ambientales como la pendiente, formación geológica asociada, altitud y cobertura vegetal. Los censos fueron agrupados mediante análisis multivariado dendrogramétrico. En total se registraron 72 especies de plantas vasculares. La fisonomía corresponde a un matorral xerófilo abierto dominado por especies de zigofiláceas y leguminosas, acompañadas por otros arbustos, cactáceas y escasas herbáceas, típicos de la provincia Fitogeográfica del Monte. A partir del análisis multivariado se detectaron cuatro unidades de vegetación relacionadas a la geología, altitud, pendiente y cobertura vegetal. Dado que este documento podría ser empleado por las autoridades encargadas de la preservación del sitio, finalmente se comentan aspectos relacionados a la conservación y uso del área.

Palabras clave: Áreas protegidas, Unidades de vegetación, Fitosociología, Desiertos hiper áridos

\section{INTRODUCCIÓN}

San Juan cuenta actualmente con 15 áreas protegidas bajo diferentes jurisdicciones, que

1 CIGEOBIO (CONICET-UNSJ) Instituto de Geología Dr. Emiliano P. Aparicio. Gabinete de Estratigrafía. Cátedra de Botánica sistemática; Departamento de Biología (FCEFyN); Universidad Nacional de San Juan. martinalmiron@conicet.gov.ar cubren el $26 \%$ de su superficie, resguardando sitios representativos de todas las provincias fitogeográficas existentes en el territorio provincial: Altoandina, Puna, Prepuna, Monte, Chaco Occidental, en sus distritos Chaco Serrano \& Chaco Árido (Márquez et al., 2017).

En algunas de estas áreas protegidas se han desarrollado estudios florísticos (Parque Nacional El Leoncito: Márquez \& Dalmasso, 2003; Reserva de la Biósfera San Guillermo: Martínez Carretero et al., 2008; Parque Provincial Ischigualasto: Márquez 
et al., 2005; Área Natural Protegida La Ciénaga: Márquez et al., 2011), que en conjunto con trabajos de otras disciplinas, permiten la elaboración de planes de manejo para la gestión y protección del patrimonio. Sin embargo, existen áreas que aún no poseen información florística, por lo que la planificación se torna dificultosa.

Mediante la Ley Provincial 7307 (2002) se creó el Monumento Natural y Paisaje Protegido Loma de las Tapias (5000 ha., aproximadamente), con el objetivo de preservar el paisaje típico de huayquerías y el yacimiento paleontológico que posee una asociación de fósiles de vertebrados de la edad MamíferoChasiquense (Plioceno Medio; Contreras, 1981), otorgando al área relevancia científica, educativa y turística. Márquez \& Pastrán (2017) describen sintéticamente la Loma de Las Tapias desde una perspectiva biogeográfica, comprendida en la Provincia Fitogeográfica del Monte y formada por un matorral de vegetación pobre y rala, principalmente vinculada a los sistemas de drenaje y a un matorral xerófilo en los sedimentos cuaternarios. Sin embargo, hasta el momento no se cuenta con relevamientos que detallen la riqueza específica del área como así tampoco de las comunidades vegetales que en ella se encuentran.

En la República Argentina, existen 32 áreas protegidas que contienen comunidades representativas del monte (Apodaca et al., 2015). A escala provincial hay 11 reservas que incluyen ambientes del monte (Márquez \& Pastrán, 2017).

Los objetivos del presente trabajo incluyen la determinación de unidades florísticas homogéneas para la obtención de la carta de vegetación y el registro de especies vegetales importantes para la conservación in situ. Finalmente se consideran aspectos a tener en cuenta en la gestión del área, ya que en ella se desarrollan numerosas actividades deportivas, recreativas y productivas (Contreras, 2010).

\section{Materiales y Métodos}

\section{Área de estudio}

El Monumento Natural y Paisaje Protegido Loma de las Tapias está ubicado a 20 kilómetros al noroeste de la Ciudad Capital de San Juan, sobre el flanco oriental de la represa de Ullum y al este y norte del cauce del río San Juan.
De acuerdo con Milana (1991) y Rodríguez (2004), la formación geológica Loma de Las Tapias está compuesta por dos miembros, denominándose "Limolitas La Colmena" al miembro inferior y "Areniscas Albardón" al superior. Sobre el oeste del área y paralelo a la megafractura Villicum-Zonda afloran los sedimentos de la Colmena, a partir de un plegamiento anticlinal erosionado (Contreras \& Baraldo, 2011). Hacia el este del plegamiento se eleva el miembro Albardón y posteriormente las formaciones Mogna y El Corral, determinadas por conglomerados (fluviales y aluviales, respectivamente). Mas al este de la Loma de Las Tapias se encuentra el plegamiento sinclinal "Las Tapias" cubierto por sedimentos cuaternarios del Holoceno (Suvires \& Contreras, 2011). En el límite sureste afloran nuevamente las formaciones Mogna y El Corral en pequeñas lomadas onduladas disectadas por cauces temporarios que desembocan en el Río San Juan. Al noroeste del área se encuentra la Loma de Ullum, donde se aprecian elementos de las formaciones Loma de Las Tapias, Mogna y El Corral, constituyentes del anticlinal detallado anteriormente.

El relieve está condicionado por diversos factores: el control tectónico dado por la interacción de las megafracturas "Villicum-Zonda" y "Salinas Grandes", la significativa erosión hídrica superficial y subsuperficial que determina un típico ambiente de huayquerías (Suvires \& Contreras, 2011) y la respuesta frente a los procesos erosivos dada por la litología y cohesión de las rocas. De este modo, quedan determinadas dos zonas elevadas: Loma de Ullum (1200 msm) y Loma de Las Tapias (1000 msm) a partir de las que se desarrollan numerosos cauces temporarios que forman cárcavas profundas donde el material es erodable.

Si bien no hay estudios edafológicos realizados dentro del área, según Liotta (2011), en las inmediaciones de la Loma de Las Tapias (Valles del Tulúm y Ullum-Zonda), los suelos son del orden Entisoles suborden Torrifluventes típicos e inmaduros, con escaso desarrollo de horizontes pedogenéticos.

De acuerdo con modelo fitoclimático de Koeppen (1931), el clima del área es del subtipo BWwkb, desértico hiper árido. La precipitación media anual es de $84 \mathrm{~mm}$, ocurriendo el $72 \%$ durante el período estival. La temperatura media anual es de $20^{\circ} \mathrm{C}$, con una media máxima de $40{ }^{\circ} \mathrm{C}$ y una media mínima de $16{ }^{\circ} \mathrm{C}$ (Poblete \& Minetti, 1999) provocando un importante déficit hídrico la mayor parte del año. 


\section{G. Almirón - Vegetación del Área Protegida "Loma de Las Tapias", San Juan}

En una síntesis de las áreas protegidas de San Juan, Márquez \& Pastrán (2017) mencionan a la Loma de las Tapias dentro de la Provincia Fitogeográfica del Monte (Cabrera, 1976), cuya vegetación corresponde en rasgos generales a un matorral de Larrea cuneifolia, Bulnesia retama, Cercidium praecox, Bougainvillea spinosa y Lycium tenuispinosum.

\section{Análisis de la vegetación}

En función de la información geológica, geomorfológica y la interpretación de imágenes satelitales, en los períodos estivales de 2014, 2015 y 2016 se realizaron 43 relevamientos de vegetación por medio del método de Braun Blanquet (1979); en parcelas de $20 \times 20 \mathrm{~m}$, se registró la cobertura aérea de todas las especies de plantas vasculares presentes. En cada relevamiento se agregó la siguiente información complementaria: pendiente del terreno $(\%)$, formación geológica en cada parcela, la cobertura vegetal general (\%), la altitud y las coordenadas geográficas. El criterio nomenclatural de los taxones botánicos fue obtenido del Catálogo de Plantas Vasculares (Zuloaga et al., 2008).

Para establecer las diferentes unidades de vegetación, se realizó un análisis multivariado dendrogramétrico de acuerdo con el método de la mínima varianza (Ward) y el cálculo de las distancias de similitud corresponde a Jaccard (Di Rienzo et al., 2016). El efecto de la altitud, formación geológica, pendiente y cobertura promedio, se analizó mediante componentes principales. Las diferentes unidades se denominaron en función de la fisonomía y la/ las especie/s dominantes.

A partir del dendrograma y de la interpretación de las variables ambientales exploradas, se elaboró la carta de vegetación (Figura 4) a escala 1: 20.000 (POSGAR 2007), empleando una imagen satelital de alta resolución (SAS-Planet, 2017). Luego de la extrapolación de las unidades, se recorrió el área constatando la correspondencia de la vegetación en los diferentes sitios.

\section{Resultados}

Se registraron 72 plantas vasculares en el área (apéndice 1). De acuerdo con el dendrograma (Figura 1) se definieron cuatro unidades de vegetación mostradas en el cuadro sintético (Tabla 1), denominadas como matorrales de:

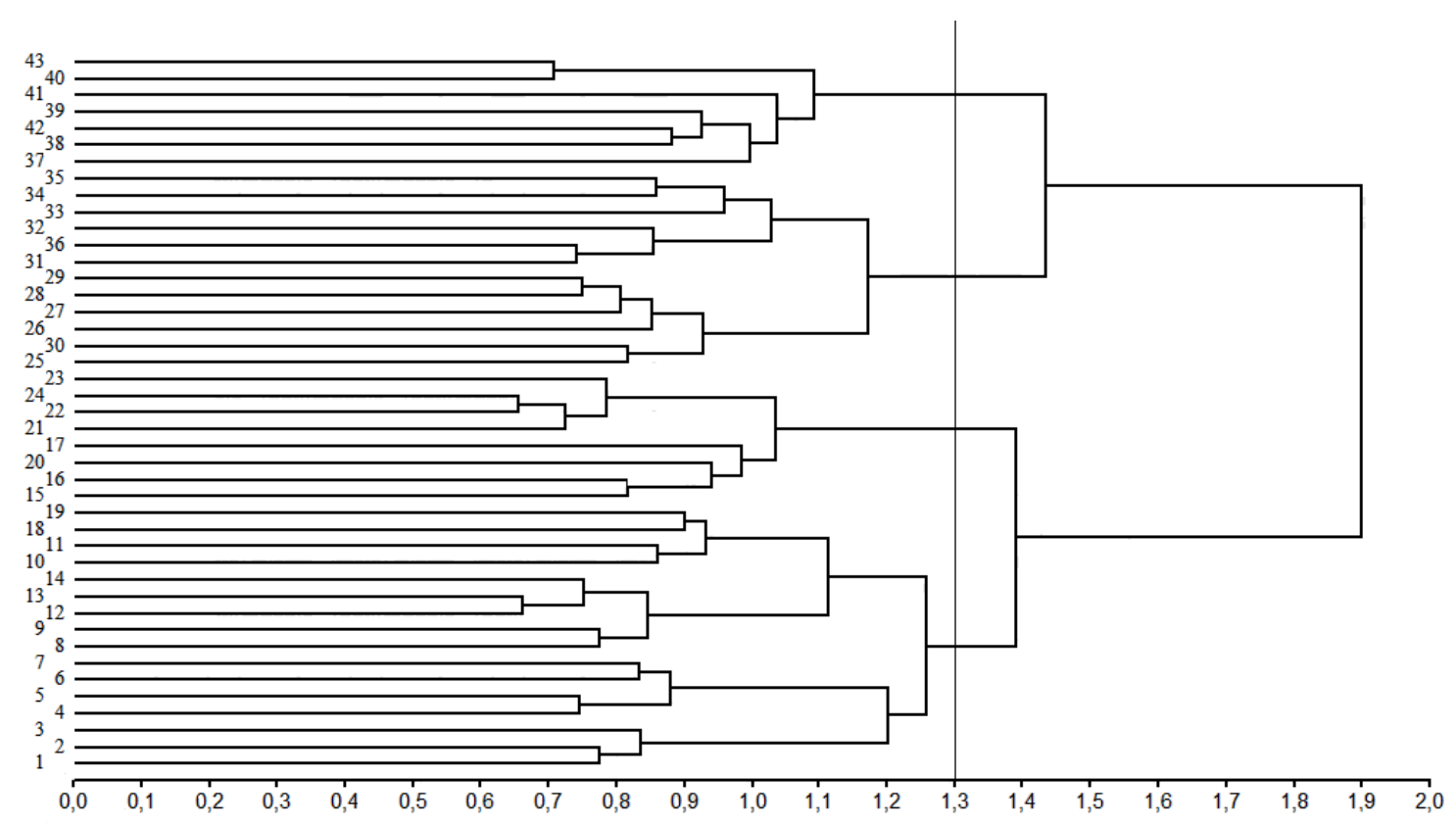

Fig. 1. Análisis dendrogramétrico de los 43 relevamientos. De abajo a arriba: Unidad 1 (Relevamientos 1-14 y 18-19); Unidad 2 (Relevamientos 15-17 y 20-24); Unidad 3 (Relevamientos 25-36) y Unidad 4 (37-43). 
Bol. Soc. Argent. Bot. 53 (3) 2018

Tabla 1. Cuadro sintético de las unidades de vegetación. Presencia (Números romanos); cobertura media (números arábigos como exponentes entre paréntesis). Otras especies en una sola unidad con (+): Unidad 1: M. ovata y J. ichu. Unidad 2: T. candicans y Z. flifolia. Unidad 3: G. cladotrichoides, P. argentina, S. eleagnifolium y S. pyramidatus. Unidad 4: C. genistioides, N. noctiflora, T. dodoneifolia y Washingtonia sp.

\begin{tabular}{|c|c|c|c|c|}
\hline Unidad & 1 & 2 & 3 & 4 \\
\hline $\mathrm{N}^{\circ}$ de relevamientos & 16 & 8 & 12 & 7 \\
\hline D. longipetala & IV $(9,1)$ & II $(2,3)$ & . & . \\
\hline La. cuneifolia & $\mathbf{V}(7,6)$ & $\mathbf{V}^{(12)}$ & III $(2,1)$ & II ${ }^{(0,4)}$ \\
\hline Z. punctata & IV $(7,2)$ & $\mathbf{V}(7,6)$ & IV $(4,3)$ & $V^{(2,5)}$ \\
\hline T. aoracanthus & $V_{(5,9)}$ & $\mathbf{V}^{(9,3)}$ & $V^{(7,9)}$ & $V^{(0,5)}$ \\
\hline B. retama & III $(3,7)$ & IV $(6,6)$ & III $(6,8)$ & IV $(1,7)$ \\
\hline L. divaricata & . & . & $\mathbf{V}^{(7,5)}$ & . \\
\hline C. praecox & IV $(3,9)$ & II $(0,8)$ & V $(5,2)$ & II $(0,9)$ \\
\hline$X$. americana & $I^{(0,5)}$ & . & IV $(4,3)$ & . \\
\hline P. flexuosa & $\mathrm{II}^{(1,0)}$ & + & III $(2,1)$ & III $(1,7)$ \\
\hline A. aroma & $I^{(0,7)}$ & . & III $(1,9)$ & III $(0,9)$ \\
\hline S. aphylla & + & + & III $(1,7)$ & III $(0,9)$ \\
\hline A. ephedroides & . & . & II $(2,4)$ & . \\
\hline Psila retamoides & . & . & II (2) & $I^{(0,4)}$ \\
\hline H. argentea & II $(0,6)$ & . & II $(1,8)$ & II $(0,4)$ \\
\hline B. colletioides & $I^{(0,5)}$ & . & II $(1,3)$ & . \\
\hline T. strigosus & $V^{(4,1)}$ & IV $(1,5)$ & II ${ }^{(+)}$ & II ${ }^{(+)}$ \\
\hline O. sulphurea & $\mathrm{V}^{(2,2)}$ & IV $(1,5)$ & III $(1,7)$ & + \\
\hline P. caespitosum & $\mathrm{V}^{(2,7)}$ & III $(2,7)$ & III $(1,4)$ & II ${ }^{(+)}$ \\
\hline E. leucantha & $\mathrm{V}^{(0,4)}$ & IV $(0,4)$ & IV $(0,8)$ & II ${ }^{(+)}$ \\
\hline A. adscencionis & IV $(2,5)$ & + & II ${ }^{(+)}$ & II ${ }^{(+)}$ \\
\hline P. sanjuanensis & IV $(1,2)$ & IV ${ }^{(+)}$ & II ${ }^{(+)}$ & . \\
\hline M. ephedroides & III $(3,5)$ & II $(2,3)$ & $I^{(0,5)}$ & + \\
\hline L. tenuispinosum & III $(2,3)$ & + & $I^{(+)}$ & $I^{(0,4)}$ \\
\hline A. incarnata & III $(0,6)$ & II ${ }^{(+)}$ & III ${ }^{(+)}$ & + \\
\hline M. parviflora & III $(0,2)$ & + & $I^{(0,3)}$ & . \\
\hline P. obscurum & III (+) & . & + & . \\
\hline B. spinosa & II $(2,2)$ & + & II $(0,5)$ & . \\
\hline A. gratissima & II $(0,6)$ & . & . & . \\
\hline T. pentachaeta & II $(0,2)$ & + & . & . \\
\hline M. albescens & II $(0,2)$ & + & + & + \\
\hline A. mendocina & II ${ }^{(+)}$ & + & III (1) & II ${ }^{(+)}$ \\
\hline E. serpens & II ${ }^{(+)}$ & . & II ${ }^{(+)}$ & . \\
\hline Lepidium sp & II ${ }^{(+)}$ & . & $\mathrm{I}^{(+)}$ & . \\
\hline
\end{tabular}


M. G. Almirón - Vegetación del Área Protegida "Loma de Las Tapias", San Juan

\begin{tabular}{|c|c|c|c|c|}
\hline Unidad & 1 & 2 & 3 & 4 \\
\hline $\mathrm{N}^{\circ}$ de relevamientos & 16 & 8 & 12 & 7 \\
\hline G. parviflora & II (+) & . & + & . \\
\hline Phacelia aff. sanzinii & II (+) & . & + & . \\
\hline C. atamisquea & $I^{(+)}$ & . & III (1) & . \\
\hline P. chilensis & + & . & III ${ }^{(0,8)}$ & $I^{(0,4)}$ \\
\hline B. aristidoides & + & . & $\mathrm{V}^{(+)}$ & . \\
\hline A. spegazzinii & $\|^{(1,6)}$ & II ${ }^{(+)}$ & II ${ }^{(0,3)}$ & $\mathbf{V}^{(2,5)}$ \\
\hline T. usillo & . & $I^{(0,4)}$ & $I^{(0,3)}$ & III $(0,9)$ \\
\hline P. tetracantha & $\left.\right|^{(0,2)}$ & + & + & III $(0,9)$ \\
\hline B. salicifolia & . & . & + & $I^{(0,4)}$ \\
\hline Li. cuneifolia & $I^{(+)}$ & . & II (+) & III ${ }^{(+)}$ \\
\hline S. kurtzii & $\left.\right|^{(0,7)}$ & . & . & II ${ }^{(+)}$ \\
\hline P. grandiflora & $I^{(+)}$ & . & || $(0,3)$ & . \\
\hline H. ameghinoi & $I^{(0,2)}$ & $I^{(1)}$ & $I^{(+)}$ & III ${ }^{(+)}$ \\
\hline P. philipianum & $I^{(+)}$ & . & $I^{(0,3)}$ & + \\
\hline C. pappophoroides & $I^{(0,5)}$ & II ${ }^{(+)}$ & + & . \\
\hline G. perackii & $\left.\right|^{(0,2)}$ & $I^{(0,4)}$ & . & . \\
\hline A. argentina & $I^{(1)}$ & . & . & . \\
\hline L. boerhaviaefolium & $I^{(0,4)}$ & . & . & . \\
\hline A. castellanosii & $I^{(0,2)}$ & . & . & . \\
\hline S. aff. pinnatus & $I^{(+)}$ & . & . & . \\
\hline G. bracteata & $I^{(+)}$ & . & . & . \\
\hline H. curassavicum & + & . & II (+) & + \\
\hline A. lampa & . & . & $I^{(0,3)}$ & + \\
\hline A. odorata & + & . & $I^{(+)}$ & . \\
\hline L. chilense & + & . & + & . \\
\hline T. crinita & + & . & . & III $\left.\right|_{(+)}$ \\
\hline T. ramossisima & . & . & + & II ${ }^{(+)}$ \\
\hline
\end{tabular}

Deuterocohnia longipetala y Larrea cuneifolia (Unidad 1), Larrea cuneifolia y Zuccagnia punctata (Unidad 2), Larrea divaricata (Unidad 3) y Atriplex spegazzinii y Zuccagnia punctata (Unidad 4).

Las variables ambientales consideradas se muestran en la Figura 2. En la Unidad 1 se observaron los mayores valores de pendiente, altitud y cobertura, seguidos por la Unidad 2. La Unidad 4 fue la más disímil, donde se observó la menor cobertura media y pendiente, aunque se encuentra altitudinalmente sobre la Unidad 3.

El análisis de componentes principales explicó el $73,7 \%$ de la varianza (factor $1: 56,9 \%$; factor $2: 16,8$ $\%$ ) (Figura 3). En el factor 1 resultaron significativas todas las variables con sus respectivos autovectores: pendiente $(-0,52)$; altitud $(-0,51)$; cobertura vegetal $(-0,50)$ y formación geológica $(0,47)$, mientras que en el factor 2 resultaron significativas la formación geológica $(0,78)$ y la pendiente $(0,51)$. 
Bol. Soc. Argent. Bot. 53 (3) 2018
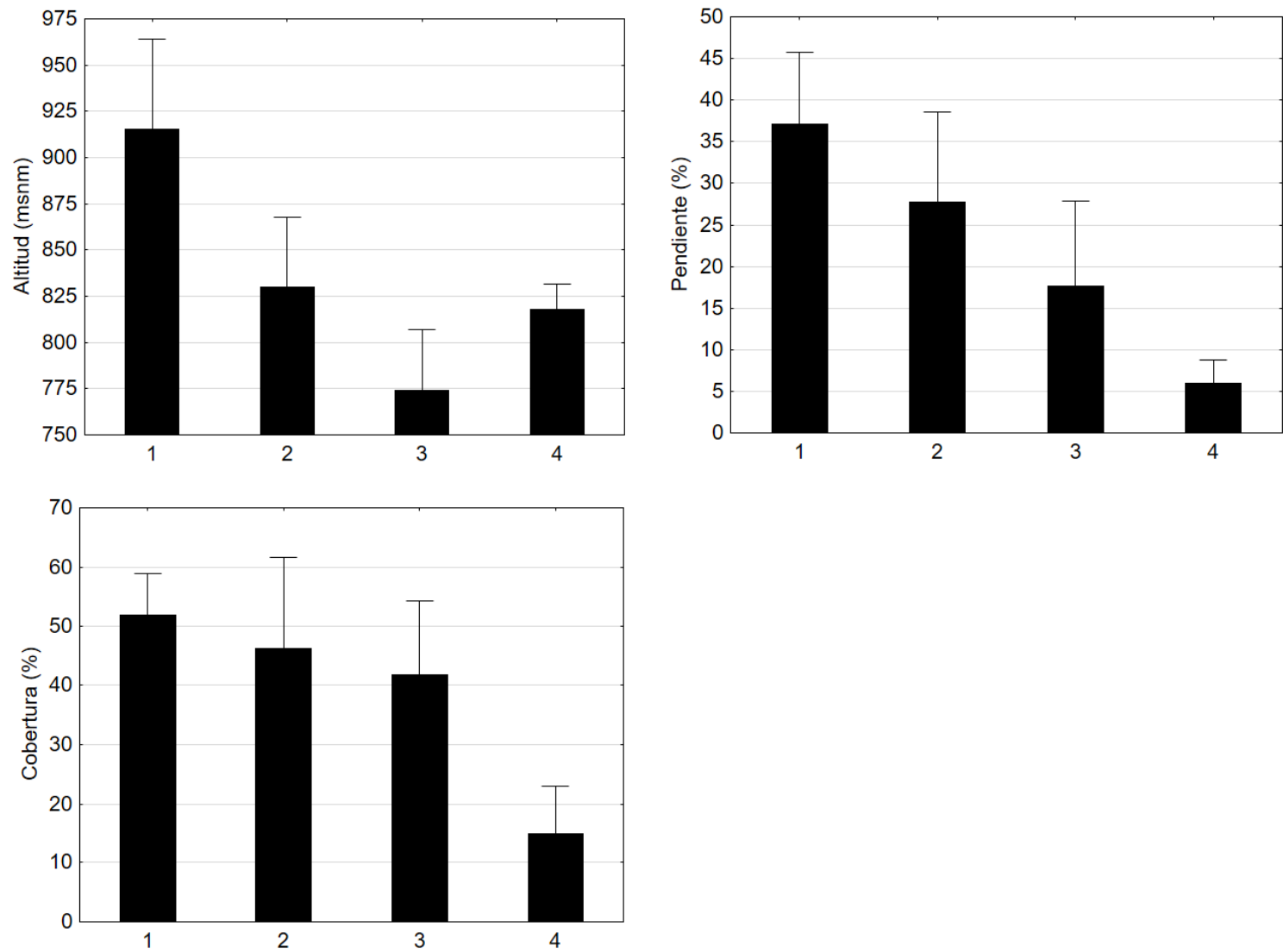

Fig. 2. Altitud, pendiente y Cobertura general en las cuatro unidades florísticas detectadas. Barras indican Desviación Standard.

A continuación, se detallan las comunidades encontradas.

Unidad 1. Matorral de Deuterocohnia longipetala y Larrea cuneifolia

(Tabla 2; relevamientos: $1-14$ y $18-19$ )

Esta comunidad está emplazada a una altura de 915,6 msnm $( \pm 90,7 \mathrm{SD})$, con pendientes abruptas $37,1 \%( \pm 16,2 \mathrm{SD})$ y coincidiendo con los afloramientos de los conglomerados Mogna y El Corral en las cimas de las Lomas de Ullum y Las Tapias. Se encontraron 55 especies con la mayor cobertura promedio $(51,9 \% ; \pm 13,1 \mathrm{SD})$, abarcando $6,7 \mathrm{~km}^{2}$ (13,5\% del área). Domina el estrato arbustivo Deuterocohnia longipetala; una especie saxícola que forma terrazas denominadas "chaguarales", que al retener sedimentos facilitan la instalación de otras especies, principalmente Larrea cuneifolia y Zuccagnia punctata.
Se observó una importante presencia de cactáceas (Tephrocactus aoracanthus, Opuntia sulphurea var. Sulphurea; Trichocereus strigosus y Pyrrhocactus sanjuanensis) con un gran número de individuos. Sobre las quebradas laterales donde se canaliza el agua la escorrentía, aumenta la presencia de Cercidium praecox. Pappophorum caespitosum y Aristida adsencionis dominan el estrato herbáceo.

Unidad 2. Matorral de Larrea cuneifolia $y$ Zuccagnia punctata

(Tabla 3; relevamientos: $15-17$ y $20-24$ )

Cubre el $13,9 \mathrm{Km}^{2}(28,2 \%$ del área) a una altitud de $830 \mathrm{msnm}( \pm 45,0 \mathrm{SD})$ sobre terrazas cuya pendiente media es $27,7 \%( \pm 13,0 \mathrm{SD})$ y que poseen una fina capa de los sedimentos provenientes de las zonas de mayor cota. Esta unidad se presenta de modo discontinuo en tres sectores principales: en el faldeo este de la Loma de Ullum, alrededor de la 
M. G. Almirón - Vegetación del Área Protegida "Loma de Las Tapias”, San Juan
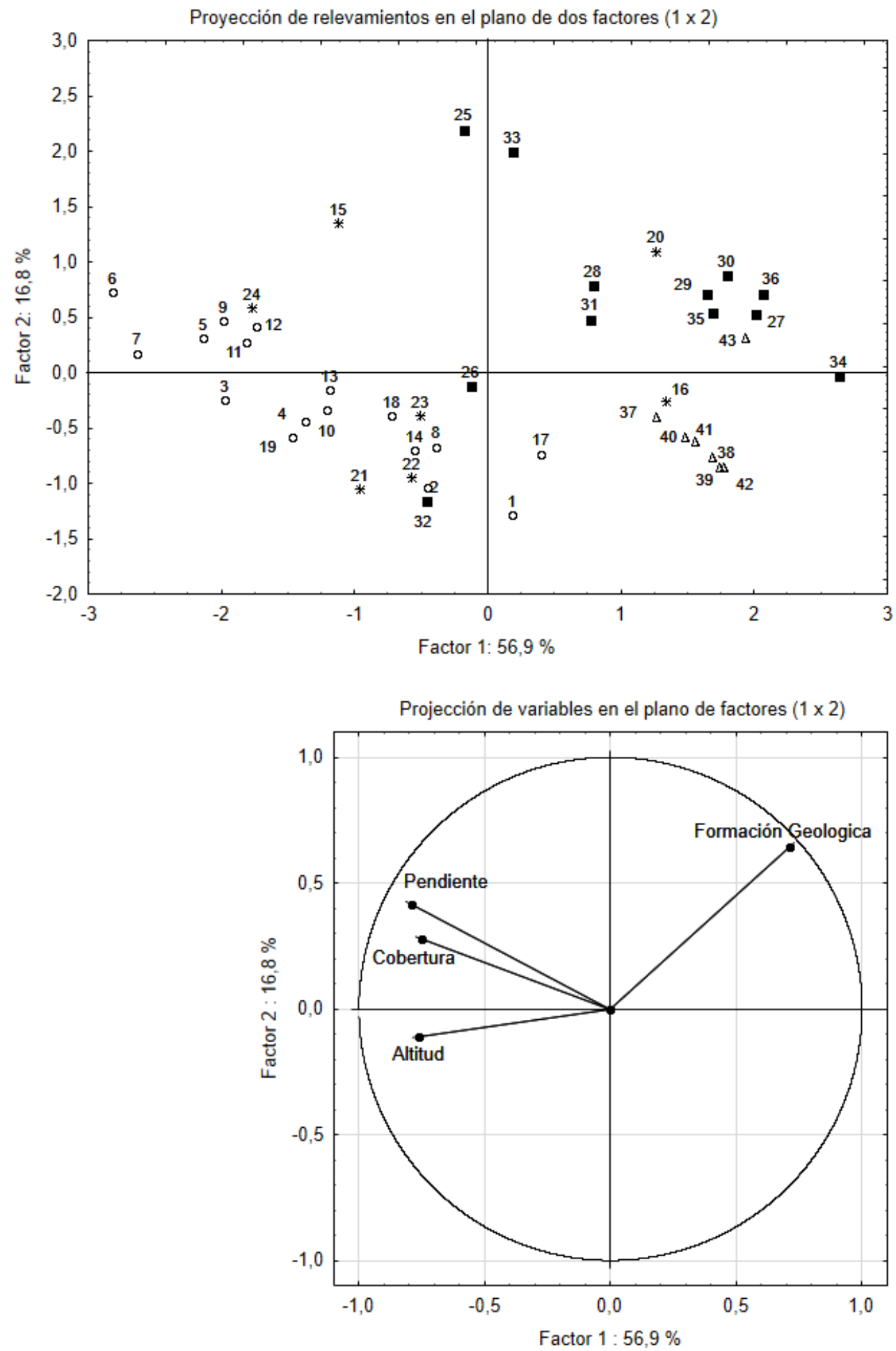

Fig. 3. Análisis de componentes principales. Arriba: Proyección de relevamientos (círculos blancos: Unidad 1; asteriscos: Unidad 2; cuadrados negros: Unidad 3; triángulos blancos: Unidad 4. Abajo: Proyección de las variables asociadas a las unidades florísticas. 
Bol. Soc. Argent. Bot. 53 (3) 2018

MONUMENTO NATURAL Y PAISAJE PROTEGIDO LOMA DE LAS TAPIAS

(Ley Provincial 7307/2002)

\section{UNIDADES DE VEGETACIÓN}

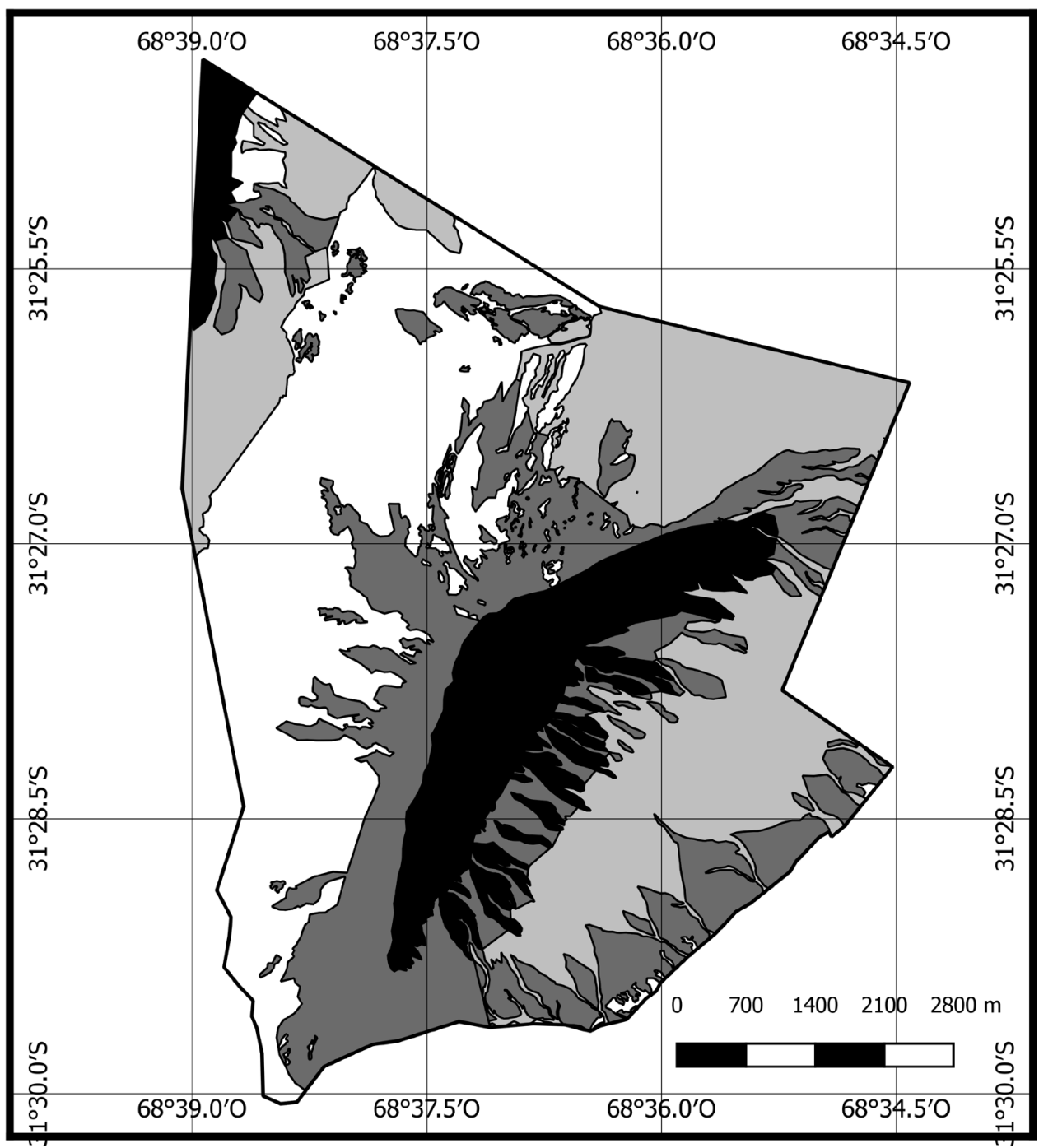

Almirón, M. (2018)

Unidades de vegetación

Deuterocohnia longipetala y Larrea cuneifolia

Larrea cuneifolia y Zuccagnia punctata

Larrea divaricata

Atriplex spegazzinii y Zuccagnia punctata

Fig. 4. Carta de Vegetación. 


\begin{tabular}{|c|c|c|c|c|c|c|c|c|c|c|c|c|c|c|c|c|c|c|}
\hline$x$ & $\begin{array}{l}\text { సे } \\
\text { ì } \\
\text { in } \\
0 \\
0 \\
0\end{array}$ & 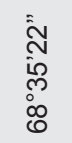 & $\begin{array}{l}0 \\
\varnothing \\
0 \\
\infty \\
\infty \\
0\end{array}$ & $\begin{array}{l}\overline{1} \\
\omega \\
\infty \\
0 \\
0 \\
\infty \\
0\end{array}$ & $\begin{array}{l}\hat{i} \\
\stackrel{1}{0} \\
\infty \\
0 \\
0 \\
\infty \\
0\end{array}$ & $\begin{array}{l}\hat{i} \\
10 \\
0 \\
0 \\
0 \\
0 \\
0\end{array}$ & 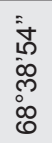 & 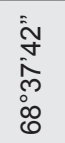 & $\begin{array}{l}\tilde{0} \\
\omega \\
\infty \\
0 \\
0 \\
\infty \\
0\end{array}$ & $\begin{array}{l}1 \\
\hat{A} \\
0 \\
\hat{N} \\
8 \\
0 \\
0\end{array}$ & 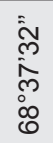 & $\begin{array}{l}\bar{y} \\
\dot{\sigma} \\
0 \\
\infty \\
\infty \\
0 \\
0\end{array}$ & $\begin{array}{l}10 \\
i \\
00 \\
00 \\
0 \\
0 \\
0\end{array}$ & 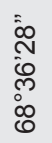 & 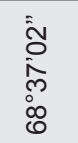 & $\begin{array}{l}\text { స̃ } \\
\stackrel{N}{\text { No }} \\
0 \\
\infty \\
0\end{array}$ & \multirow{7}{*}{$\begin{array}{l}. \frac{\pi}{0} \\
\frac{\tilde{0}}{0} \\
0 \\
0 \\
0 \\
0\end{array}$} & \multirow{7}{*}{$\begin{array}{l} \\
0 \\
\circ \\
\varnothing \\
0 \\
0 \\
0 \\
0 \\
0\end{array}$} \\
\hline Y & 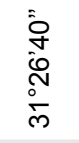 & 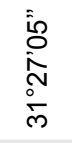 & $\begin{array}{l}\dot{\bar{d}} \\
\text { ì } \\
\text { o } \\
\bar{m}\end{array}$ & 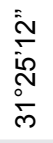 & $\begin{array}{l}\bar{i} \\
\dot{0} \\
\dot{N} \\
\dot{N} \\
\dot{m}\end{array}$ & 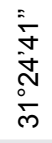 & 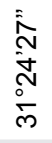 & $\begin{array}{l}\bar{\Sigma} \\
\dot{\Sigma} \\
\stackrel{N}{\circ} \\
\dot{m}\end{array}$ & 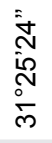 & $\begin{array}{l}\text { के } \\
O_{0} \\
\text { o } \\
\text { o } \\
\bar{m}\end{array}$ & 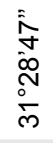 & $\begin{array}{l}\text { ò } \\
\text { o } \\
\text { N } \\
\text { o } \\
\bar{m}\end{array}$ & $\begin{array}{l}\bar{i} \\
\stackrel{N}{N} \\
\stackrel{N}{\circ} \\
\bar{n}\end{array}$ & 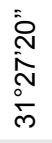 & $\begin{array}{l}\overline{\text { oे }} \\
\text { ळे } \\
\text { సे } \\
\bar{m}\end{array}$ & 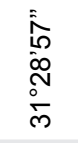 & & \\
\hline Altitud (msnm) & $\hat{\kappa}$ & $\stackrel{\infty}{\curvearrowright}$ & 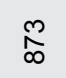 & ळे & ণั & సิ & $\begin{array}{l}\text { ஜి } \\
\stackrel{0}{\circ}\end{array}$ & ஓా & $\bar{\sigma}$ & \& & 웅 & চ̊ & ద్రి & ণ్రి & $\frac{d}{2}$ & $\underset{\infty}{\mathbb{N}}$ & & \\
\hline Cobertura (\%) & 40 & 50 & 70 & 60 & 40 & 50 & 70 & 50 & 60 & 40 & 65 & 70 & 25 & 40 & 50 & 50 & & \\
\hline Pendiente (\%) & 15 & 20 & 40 & 20 & 60 & 70 & 40 & 13 & 50 & 35 & 40 & 40 & 50 & 20 & 40 & 40 & & \\
\hline Fm. Geológica & E.C. & E.C. & E.C. & M. & M. & M. & M. & M. & M. & M. & M. & M. & M. & M. & E.C. & E.C. & & \\
\hline Relevamiento & 1 & 2 & 3 & 4 & 5 & 6 & 7 & 8 & 9 & 10 & 11 & 12 & 13 & 14 & 18 & 19 & & \\
\hline D. longipetala & & & & 3 & 3 & 8 & 18 & 18 & 8 & + & 38 & & 8 & 38 & & 3 & IV & 9,07 \\
\hline La. cuneifolia & 18 & 8 & & 3 & 3 & 3 & & 8 & 8 & 8 & 8 & 18 & 8 & 18 & 3 & 8 & V & 7,63 \\
\hline Z. punctata & 18 & 8 & 8 & 3 & 8 & 8 & 18 & 3 & & 3 & & + & 38 & & & & IV & 7,19 \\
\hline T. aoracanthus & 3 & 3 & 3 & 8 & 3 & 3 & & 3 & 8 & 18 & 3 & 8 & 8 & 8 & 8 & 8 & v & 5,94 \\
\hline T. strigosus & & & + & 3 & 3 & 3 & 3 & 3 & 8 & 3 & 8 & 8 & 8 & 8 & + & 8 & V & 4,14 \\
\hline C. praecox & 3 & 8 & 3 & 3 & 3 & & & & & 8 & & 3 & 3 & 3 & 18 & 8 & IV & 3,94 \\
\hline B. retama & 18 & & 18 & & & & & 3 & 3 & 3 & & 3 & & 3 & 8 & & III & 3,69 \\
\hline M. ephedroides & 8 & 18 & & & & & & 8 & 8 & 3 & & 3 & 8 & & + & & III & 3,51 \\
\hline P. caespitosum & + & & + & + & 3 & + & 3 & & 3 & + & + & 8 & 18 & 8 & + & & V & 2,73 \\
\hline A. adscencionis & 3 & 3 & + & 8 & 3 & & 8 & + & 3 & + & & 8 & 3 & & + & & IV & 2,46 \\
\hline L. tenuispinosum & 3 & 8 & 8 & & 3 & & & & & 3 & & 3 & & 8 & & & III & 2,25 \\
\hline O. sulphurea & + & & 3 & 3 & 3 & 3 & + & 3 & 3 & 3 & + & 8 & 3 & 3 & + & & V & 2,21 \\
\hline B. spinosa & 3 & 3 & 18 & & 3 & & & & & 8 & & & & & & & II & 2,19 \\
\hline A. spegazzinii & & 3 & & 3 & 8 & 8 & 3 & & & & & & & & & & II & 1,56 \\
\hline P. sanjuanensis & & & & + & + & & 3 & 3 & 3 & & + & 3 & + & 3 & 3 & + & IV & 1,16 \\
\hline P. flexuosa & & 8 & 8 & + & & + & & & & & & & & & & & ॥ & 1,01 \\
\hline A. argentina & & & & & + & 8 & 8 & & & & & & & & & & I & 1,01 \\
\hline S. kurtzii & & & & 8 & 3 & + & & & & & & & & & & & 1 & 0,69 \\
\hline A. aroma & & 3 & & & & & & & & 8 & & & & & + & & I & 0,69 \\
\hline A. incarnata & & & & 3 & + & + & 3 & & + & + & & + & & & + & 3 & III & 0,60 \\
\hline A. gratissima & & & & 3 & & & 3 & & 3 & & + & & & & & & II & 0,57 \\
\hline H. argentea & & & + & & 3 & & & & & 3 & & & & & 3 & & II & 0,57 \\
\hline C. pappophoroides & & & & & + & & & & & & & 8 & + & & & & 1 & 0,51 \\
\hline X. americana & 8 & & & & & & & & & & & & & & & & 1 & 0,50 \\
\hline B. colletioides & & & & & & & & & & & & & & & 8 & & I & 0,50 \\
\hline E. leucantha & + & & + & + & + & & & + & + & + & + & + & + & + & 3 & 3 & V & 0,44 \\
\hline L. boerhaviaefolium & & & & 3 & & 3 & & & & & & & & & & & 1 & 0,38 \\
\hline M. parviflora & & & + & & + & & & & & + & & + & + & 3 & & + & III & 0,23 \\
\hline M. albescens & & & & & & + & & + & & + & + & & & & 3 & & II & 0,21 \\
\hline T. pentachaeta & & & & & & & & & + & + & 3 & & & & + & & II & 0,21 \\
\hline
\end{tabular}




\begin{tabular}{|c|c|c|c|c|c|c|c|c|c|c|c|c|c|c|c|c|c|c|}
\hline$x$ & 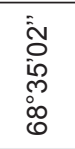 & 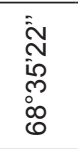 & $\begin{array}{l}\bar{D} \\
\dot{0} \\
0 \\
ల \\
0 \\
0 \\
0\end{array}$ & $\begin{array}{l}\bar{u} \\
\omega \\
\infty \\
\infty \\
\infty \\
\infty \\
0 \\
0\end{array}$ & $\begin{array}{l}\hat{i} \\
\stackrel{1}{0} \\
\infty \\
m \\
\infty \\
\infty \\
0\end{array}$ & $\begin{array}{l}\hat{i} \\
10 \\
\infty \\
\infty \\
\infty \\
\infty \\
0\end{array}$ & 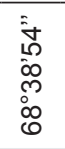 & 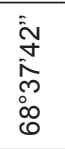 & 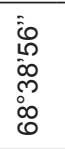 & $\begin{array}{l}\hat{i} \\
\hat{i} \\
0 \\
o \\
0 \\
0\end{array}$ & 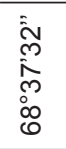 & 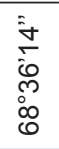 & $\begin{array}{l}i \\
i \\
0 \\
0 \\
\infty \\
0 \\
0 \\
0\end{array}$ & 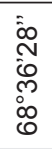 & 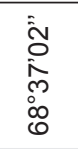 & $\begin{array}{l}\text { స̃ } \\
\stackrel{\hat{N}}{o} \\
0 \\
0 \\
0\end{array}$ & \multirow{7}{*}{$\begin{array}{l}\frac{\pi}{0} \\
\frac{D}{D} \\
\mathbb{D} \\
0 \\
0\end{array}$} & \multirow{7}{*}{ 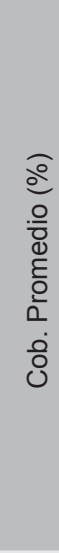 } \\
\hline Y & 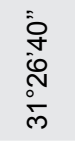 & 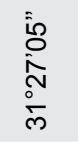 & $\begin{array}{l}\bar{d} \\
\text { Dे } \\
\text { Ǹ} \\
\bar{\delta} \\
\bar{m}\end{array}$ & 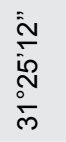 & 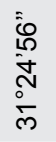 & $\begin{array}{l}\bar{y} \\
\bar{J} \\
\dot{N} \\
\frac{N}{m}\end{array}$ & $\begin{array}{l}\stackrel{D}{N} \\
\stackrel{+}{N} \\
\stackrel{N}{\infty}\end{array}$ & 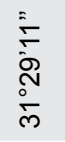 & $\begin{array}{l}\bar{i} \\
\stackrel{i}{N} \\
\stackrel{N}{N} \\
\stackrel{N}{m}\end{array}$ & 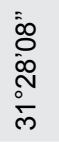 & $\begin{array}{l}\bar{i} \\
\text { jo } \\
\text { o } \\
\text { N } \\
\bar{m}\end{array}$ & $\begin{array}{l}\text { ò } \\
\text { o } \\
\text { N } \\
\text { ò } \\
\bar{m}\end{array}$ & 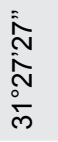 & 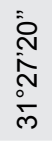 & 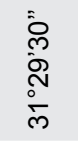 & 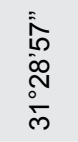 & & \\
\hline Altitud (msnm) & h & $\stackrel{\infty}{\circ}$ & $\underset{\infty}{\stackrel{\infty}{\infty}}$ & চ্ণ & ̊̊ & సิ & 욤 & ১্ & ॄे & \& & 오 & \& & 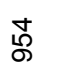 & ద్లి & 寺 & $\underset{\infty}{\mathbb{N}}$ & & \\
\hline Cobertura (\%) & 40 & 50 & 70 & 60 & 40 & 50 & 70 & 50 & 60 & 40 & 65 & 70 & 25 & 40 & 50 & 50 & & \\
\hline Pendiente (\%) & 15 & 20 & 40 & 20 & 60 & 70 & 40 & 13 & 50 & 35 & 40 & 40 & 50 & 20 & 40 & 40 & & \\
\hline Fm. Geológica & E.C. & E.C. & E.C. & M. & M. & M. & M. & M. & M. & M. & M. & M. & M. & M. & E.C. & E.C. & & \\
\hline Relevamiento & 1 & 2 & 3 & 4 & 5 & 6 & 7 & 8 & 9 & 10 & 11 & 12 & 13 & 14 & 18 & 19 & & \\
\hline A. castellanosii & & & & & & & & & & & 3 & & & + & & & I & 0,19 \\
\hline G. perackii & & & & & & & & & & & + & 3 & & & & & I & 0,19 \\
\hline H. ameghinoi & & & & & & 3 & & & & & & & & & & & 1 & 0,19 \\
\hline P. tetracantha & & & & & & 3 & & & & & & & & & & & I & 0,19 \\
\hline P. obscurum & + & & + & + & + & & & & & + & & + & + & + & + & & III & 0,06 \\
\hline A. mendocina & & & & + & + & & & + & & + & + & & & & + & & II & 0,04 \\
\hline P. aff. sanzinii & & & & + & + & + & + & & + & & & & & & & & II & 0,03 \\
\hline G. parviflora & & & & & & & + & & & + & + & + & + & & & & II & 0,03 \\
\hline Lepidium $s p$ & & & & + & + & + & & & + & & & & & & & & II & 0,03 \\
\hline E. serpens & & & + & & & & + & & + & & & & & & & + & II & 0,03 \\
\hline S. aff. pinnatus & & & & & & + & & & & & + & & + & & & & 1 & 0,02 \\
\hline G. bracteata & & & & + & & + & + & & & & & & & & & & I & 0,02 \\
\hline C. atamisquea & & & + & & & & & & + & & & & & & & & 1 & 0,01 \\
\hline Li. cuneifolia & & & & & & & & & & + & + & & & & & & 1 & 0,01 \\
\hline P. philipianum & & & & & & & & & & & + & & & & + & & 1 & 0,01 \\
\hline P. grandiflora & + & & & & & & & & & & & & & & + & & 1 & 0,01 \\
\hline
\end{tabular}

loma de Las Tapias y sobre el límite sureste del área (Fm. Mogna y El Corral). En el faldeo noroeste de la loma de Las Tapias se encuentra muy disectada por los procesos de erosión hídrica que exponen las areniscas neógenas del miembro Albardón, por lo que el paisaje corresponde a un mosaico alternando y discontinuo con la vegetación típica de la unidad 1 .

Se encontraron 30 especies. La cobertura alcanza el 46,2 \% ( $\pm 18,5 \mathrm{SD})$, dominado por Larrea cuneifolia, Zuccagnia punctata y Bulnesia retama. Las cactáceas son también muy frecuentes, siendo las opuntioideas las principales dominantes (Tephrocactus aoracanthus y Opuntia suphurea var. Sulphurea), mientras que las cactoideas (Trichocereus strigosus, Pyrrhocactus sanjuanensis y Echinopsis leucantha) son frecuentes, aunque con menor cobertura y casi siempre encontradas bajo la canopia de los arbustos. En el estrato herbáceo, sólo se destaca Pappophorum caespitosum.

Unidad 3. Matorral de Larrea divaricata

(Tabla 4; relevamientos: 25-36)

Se asienta en faldeos suaves de menor altitud (774,0 $\mathrm{msnm} \pm 51,7 \mathrm{SD}$ ) formados por depósitos coluviales-aluviales modernos del Holoceno, provenientes de las zonas elevadas laterales. Esta unidad se observó en tres sectores (este; noreste $\mathrm{y}$ noroeste) disectados por numerosos cauces temporarios en un relieve ondulado con una pendiente promedio de $17,5 \%( \pm 16,1 \mathrm{SD})$. La superficie abarca 14,6 $\mathrm{Km}^{2}$ (29,5\% del área). El sector ubicado al este se dispone sobre los depósitos modernos que cubren el plegamiento sinclinal "Las Tapias", drenando en el río San Juan. El sector 
Tabla 3. Matorral de Larrea cuneifolia y Z. punctata. Valores de cobertura (\%). Otras especies halladas en un solo relevamiento con cobertura inferior al $1 \%$ : A. adsencionis, M. albescens, S. aphylla, $T$. candicans (Relev. 16); P. flexuosa, B. spinosa (Relev. 17); P. tetracantha, Z. filifolia (Relev. 21); L. tenuispinosum, M. parviflora, T. pentachaeta (Relev. 22); A. mendocina (Relev. 23). Fm. Geológica: Albardón (Alb.); Holoceno (Holoc.); El Corral (E.C.); Mogna (M.)

\begin{tabular}{|c|c|c|c|c|c|c|c|c|c|c|}
\hline$x$ & $\begin{array}{l}\bar{c} \\
\overline{0} \\
\infty \\
0 \\
\infty \\
\infty \\
0\end{array}$ & $\begin{array}{l}0 \\
\varnothing \\
\infty \\
\infty \\
\infty \\
\infty\end{array}$ & 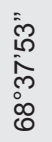 & $\begin{array}{l}\text { సे } \\
\text { iे } \\
\text { in } \\
\infty \\
\infty \\
0\end{array}$ & $\begin{array}{l}\overline{0} \\
\dot{+} \\
\infty \\
0 \\
o \\
0 \\
0\end{array}$ & $\begin{array}{l}\bar{y} \\
\text { ले } \\
\text { in } \\
\infty \\
\infty \\
0\end{array}$ & 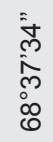 & $\begin{array}{l}\text { iे } \\
\text { iv } \\
\text { o } \\
0 \\
0 \\
0\end{array}$ & \multirow{7}{*}{ 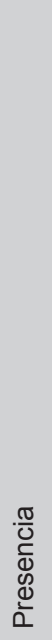 } & \multirow{7}{*}{ 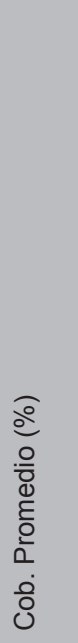 } \\
\hline Y & 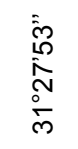 & 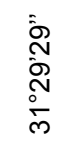 & 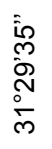 & 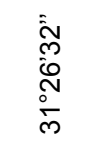 & 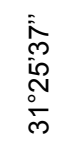 & 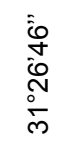 & $\begin{array}{l}\infty \\
\stackrel{\infty}{\infty} \\
\stackrel{N}{N} \\
\stackrel{\infty}{\circ}\end{array}$ & 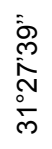 & & \\
\hline Altitud (msnm) & $\stackrel{\text { क़ }}{\infty}$ & হ & స్ & 吕 & $\underset{\infty}{\infty}$ & $\underset{\infty}{\mp}$ & đ్ & $\begin{array}{l}\stackrel{0}{\infty} \\
\infty\end{array}$ & & \\
\hline Cobertura (\%) & 70 & 20 & 40 & 40 & 40 & 60 & 70 & 70 & & \\
\hline Pendiente (\%) & 45 & 15 & 12 & 30 & 30 & 15 & 45 & 45 & & \\
\hline Fm. Geológica & Alb. & Alb. & M. & Holoc. & E.C. & E.C. & M. & M. & & \\
\hline Relevamiento & 15 & 16 & 17 & 20 & 21 & 22 & 23 & 24 & & \\
\hline La. cuneifolia & 18 & 8 & 3 & + & 8 & 18 & 38 & 3 & V & 12,01 \\
\hline T. aoracanthus & 18 & 8 & 3 & 18 & 8 & 8 & 3 & 8 & v & 9,25 \\
\hline Z. punctata & 3 & 8 & 8 & + & 8 & 18 & 8 & 8 & V & 7,64 \\
\hline B. retama & & 3 & & 8 & 8 & 18 & 8 & 8 & V & 6,63 \\
\hline P. caespitosum & & & & & 3 & + & 18 & + & III & 2,65 \\
\hline D. longipetala & & + & 18 & & & + & & & II & 2,28 \\
\hline M. ephedroides & & & + & & + & & 18 & & II & 2,28 \\
\hline T. strigosus & & + & + & & 3 & 3 & 3 & 3 & V & 1,53 \\
\hline O. sulphurea & 3 & + & 3 & & & 3 & 3 & & IV & 1,51 \\
\hline H. ameghinoi & & & & 8 & & & & & 1 & 1,00 \\
\hline C. praecox & & + & 3 & & & & & 3 & II & 0,76 \\
\hline E. leucantha & + & + & 3 & + & & + & + & & V & 0,44 \\
\hline G. perackii & & & & & & & 3 & & 1 & 0,38 \\
\hline T. usillo & & & & & 3 & & & & 1 & 0,38 \\
\hline P. sanjuanensis & + & + & & & + & + & + & & IV & 0,06 \\
\hline A. incarnata & & + & & & + & + & & & ॥ & 0,04 \\
\hline A. spegazzinii & & + & + & & & & & & II & 0,03 \\
\hline C. pappophoroides & & & & & & & + & + & II & 0,03 \\
\hline
\end{tabular}

noroeste, se ubica sobre los abanicos aluviales de la Loma de Ullum que drena hacia la presa homónima, mientras que el sector noreste descarga en el río temporario Dávila.

La cobertura vegetal es de $41,7 \%( \pm 20,0 \mathrm{SD})$, con 53 especies. Corresponde a arbustales de Larrea divaricata, Bulnesia retama y Zuccagnia punctata en los interfluvios, mientras que en los márgenes de los cauces temporarios dominan Cercidium praecox, Prosopis flexuosa y Ximenia americana. En los cauces temporarios, sobre pequeños domos de material retenido por la vegetación, dominan Acacia 
Bol. Soc. Argent. Bot. 53 (3) 2018

Tabla 4. Matorral de Larrea divaricata. Valores de cobertura (\%). Otras especies halladas en un solo

relevamiento con cobertura inferior al 1\%: P. obscurum, M. albescens, L. chilense (Relev. 25); G.

cladotrichoides (Relev. 27); T. ramossisima, P. aff. sanzinii (Relev. 31); B. salicifolia, P. argentina, S.

eleagnifolium, S. pyramidatus (Relev. 33); P. tetracantha (Relev. 34); G. parviflora C. pappophoroides (Relev. 35). Fm. Geológica: Holoceno (H.); El Corral (E.C.); Mogna (M.).

\begin{tabular}{|c|c|c|c|c|c|c|c|c|c|c|c|c|c|c|}
\hline$x$ & $\begin{array}{l}1 \\
\hat{O} \\
\text { o } \\
0 \\
\infty \\
0\end{array}$ & $\begin{array}{l}\overline{0} \\
\frac{0}{\phi} \\
\infty \\
\infty \\
0\end{array}$ & $\begin{array}{l}\text { o } \\
\text { oे } \\
\dot{~} \\
\text { oे } \\
\infty \\
0\end{array}$ & $\begin{array}{l}\overline{0} \\
\text { ơ } \\
\infty \\
0 \\
0 \\
0\end{array}$ & $\begin{array}{l}\hat{\hat{N}} \\
\text { in } \\
\text { in } \\
\infty \\
\infty \\
0\end{array}$ & $\begin{array}{l}\hat{i} \\
\text { in } \\
\text { లె } \\
\infty \\
\infty \\
0\end{array}$ & $\begin{array}{l}\hat{0} \\
0 \\
0 \\
0 \\
\infty \\
0\end{array}$ & 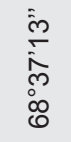 & $\begin{array}{l}\tilde{\text { in }} \\
\text { in } \\
\text { లె } \\
\infty \\
\infty \\
0\end{array}$ & 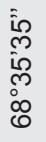 & $\begin{array}{l}\text { in } \\
\text { ஸे } \\
\text { o } \\
\infty \\
0\end{array}$ & 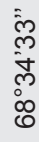 & \multirow{7}{*}{$\begin{array}{l}\frac{\pi}{0} \\
\frac{E}{\Phi} \\
\mathbb{D} \\
\stackrel{0}{L}\end{array}$} & \multirow{7}{*}{ 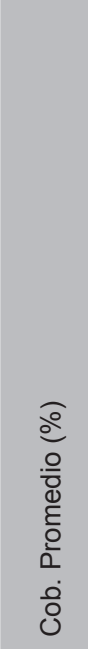 } \\
\hline Y & $\begin{array}{l}\bar{\omega} \\
\text { o } \\
o \\
\stackrel{N}{o} \\
\bar{m}\end{array}$ & 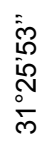 & 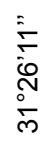 & 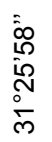 & 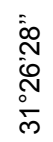 & $\begin{array}{l}\bar{D} \\
\text { Oे } \\
\text { के } \\
\text { o } \\
\bar{m}\end{array}$ & $\begin{array}{l}\bar{\alpha} \\
\text { ò } \\
\dot{\Delta} \\
\stackrel{+}{\circ} \\
\bar{n}\end{array}$ & $\begin{array}{l}\bar{\nabla} \\
\overline{\text { Dे }} \\
\text { o } \\
\bar{n}\end{array}$ & $\begin{array}{l}\overline{0} \\
\text { in } \\
\text { in } \\
\text { o } \\
\bar{m}\end{array}$ & $\begin{array}{l}\overline{0} \\
\overline{0} \\
\stackrel{0}{0} \\
\bar{\delta}\end{array}$ & $\begin{array}{l}\overline{0} \\
\text { in } \\
\text { in } \\
\text { o } \\
\bar{m}\end{array}$ & 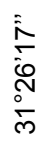 & & \\
\hline Altitud (msnm) & 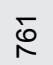 & $\underset{N}{\mathbb{N}}$ & $\stackrel{\mathbb{N}}{\mathbb{N}}$ & $\underset{\infty}{\infty}$ & 疋 & $\stackrel{\mathscr{N}}{\sim}$ & চ্் & న్ & 员 & $\stackrel{5}{n}$ & $\frac{R}{R}$ & $\stackrel{10}{i}$ & & \\
\hline Cobertura (\%) & 60 & 40 & 60 & 40 & 15 & 40 & 60 & 50 & 5 & 40 & 20 & 30 & & \\
\hline Pendiente (\%) & 20 & 2 & 5 & 10 & 30 & 10 & 10 & 50 & 5 & 5 & 20 & 5 & & \\
\hline Fm. Geológica & $\mathrm{H}$. & M. & $\mathrm{H}$. & $\mathrm{H}$. & $\mathrm{H}$. & $\mathrm{H}$. & $\mathrm{H}$. & E.C. & $\mathrm{H}$. & $\mathrm{H}$. & $\mathrm{H}$. & $\mathrm{H}$. & & \\
\hline Relevamiento & 25 & 26 & 27 & 28 & 29 & 30 & 31 & 32 & 33 & 34 & 35 & 36 & & \\
\hline L. divaricata & 3 & 8 & 18 & 8 & 8 & 18 & 3 & 3 & & & 3 & 18 & IV & 7,50 \\
\hline T. aoracanthus & 8 & 18 & 8 & 18 & 8 & 18 & 8 & 3 & & 3 & & 3 & IV & 7,92 \\
\hline B. retama & 18 & 3 & 8 & & 8 & 38 & 3 & & & & 3 & & III & 6,75 \\
\hline C. praecox & + & 3 & 8 & 8 & 8 & 3 & 8 & 18 & + & 3 & & 3 & V & 5,18 \\
\hline Z. punctata & & 8 & & 8 & 18 & & 3 & 3 & 3 & 8 & + & & IV & 4,26 \\
\hline X. americana & & + & & 8 & 8 & 8 & 3 & & & 8 & 8 & 8 & III & 4,26 \\
\hline La. cuneifolia & 8 & 3 & & 8 & & 3 & 3 & & & + & + & & III & 3,77 \\
\hline A. ephedroides & & & & & & & & & & 3 & 8 & 18 & I & 2,42 \\
\hline P. flexuosa & + & 3 & & & 8 & & 3 & & & 8 & & 3 & III & 2,09 \\
\hline P. retamoides & & & & & & & 8 & & + & & 8 & 8 & ॥ & 2,01 \\
\hline A. aroma & & & & & + & 3 & 8 & 8 & + & & + & 3 & III & 1,86 \\
\hline H. argentea & & & & & & & 3 & 8 & & & 3 & 8 & II & 1,83 \\
\hline S. aphylla & & & 3 & + & 3 & & 3 & 8 & & + & 3 & & III & 1,68 \\
\hline O. sulphurea & 8 & 3 & & 3 & 3 & 3 & + & & & & & & III & 1,68 \\
\hline P. caespitosum & 3 & & & & & 3 & 3 & + & & & & 8 & II & 1,43 \\
\hline B. colletioides & & & & & & & 8 & & + & & & 8 & I & 1,34 \\
\hline C. atamisquea & & + & + & + & + & & + & & & 8 & 3 & & III & 0,96 \\
\hline A. mendocina & & + & & 3 & + & & + & + & & 8 & & & III & 0,95 \\
\hline E. leucantha & 3 & + & 3 & 3 & + & + & + & & & + & & + & IV & 0,80 \\
\hline P. chilensis & & + & + & & & & & & 3 & 3 & 3 & & III & 0,77 \\
\hline B. spinosa & & 3 & & + & & & & & & & 3 & & II & 0,51 \\
\hline M. ephedroides & & & & & & & 3 & 3 & & & & & I & 0,50 \\
\hline P. grandiflora & & 3 & + & & & + & & & & & + & & II & 0,28 \\
\hline A. spegazzinii & & 3 & + & & & & + & & & & & & II & 0,27 \\
\hline
\end{tabular}




\begin{tabular}{|c|c|c|c|c|c|c|c|c|c|c|c|c|c|c|}
\hline$x$ & $\begin{array}{l}1 \\
0 \\
0 \\
0 \\
\infty \\
\infty \\
0\end{array}$ & $\begin{array}{l}\bar{y} \\
\text { 응 } \\
0 \\
\infty \\
0 \\
0\end{array}$ & $\begin{array}{l}\text { o) } \\
\text { o } \\
\text { \&े } \\
\text { o } \\
\infty \\
0\end{array}$ & $\begin{array}{l}\bar{o} \\
\text { ơ } \\
\infty \\
\text { o } \\
\infty \\
0\end{array}$ & $\begin{array}{l}\overline{1} \\
\text { O } \\
\text { in } \\
\text { లn } \\
\infty \\
0\end{array}$ & $\begin{array}{l}\mathcal{N} \\
\text { N } \\
i \\
\infty \\
\infty \\
0 \\
0\end{array}$ & $\begin{array}{l}\hat{0} \\
\stackrel{0}{\infty} \\
m \\
\infty \\
\infty\end{array}$ & $\begin{array}{l}\tilde{c} \\
\stackrel{\hat{\lambda}}{o} \\
o \\
\infty \\
0\end{array}$ & 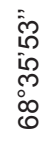 & $\begin{array}{l}i n \\
\text { in } \\
i \infty \\
\infty \\
\infty \\
\infty \\
0\end{array}$ & $\begin{array}{l}i \\
\text { in } \\
0 \\
\text { o } \\
0 \\
0 \\
0\end{array}$ & $\begin{array}{l}\overline{\text { ले }} \\
\text { m } \\
\dot{m} \\
\infty \\
\infty \\
0\end{array}$ & \multirow{7}{*}{$\begin{array}{l}\frac{\pi}{0} \\
\frac{C}{0} \\
\mathbb{D} \\
\Phi \\
0\end{array}$} & \multirow{7}{*}{ 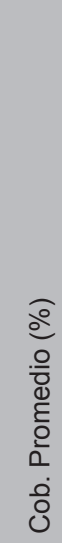 } \\
\hline $\mathrm{Y}$ & 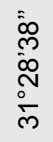 & 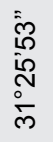 & 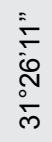 & $\begin{array}{l}\text { iे } \\
\text { in } \\
\text { in } \\
\text { o } \\
\text { n }\end{array}$ & 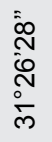 & $\begin{array}{l}\bar{y} \\
\text { o } \\
\infty \\
\text { ते } \\
\text { চ }\end{array}$ & $\begin{array}{l}\dot{\alpha} \\
\stackrel{+}{+} \\
\stackrel{+}{+} \\
\stackrel{\circ}{\infty}\end{array}$ & $\begin{array}{l}\dot{\nabla} \\
\bar{O} \\
\stackrel{\circ}{\delta} \\
\bar{m}\end{array}$ & 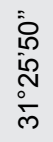 & 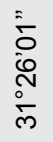 & 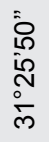 & $\begin{array}{l}\hat{i} \\
\stackrel{i}{0} \\
\stackrel{N}{\circ} \\
\bar{n}\end{array}$ & & \\
\hline Altitud (msnm) & $\bar{\phi}$ & $\stackrel{N}{\AA}$ & $\stackrel{\mathbb{N}}{\stackrel{2}{ }}$ & $\underset{\infty}{\infty}$ & 疋 & $\stackrel{\mathscr{N}}{\sim}$ & চ্ & స్ & 只 & $\stackrel{\hat{n}}{\sim}$ & $\stackrel{\circ}{N}$ & $\frac{n}{\Gamma}$ & & \\
\hline Cobertura (\%) & 60 & 40 & 60 & 40 & 15 & 40 & 60 & 50 & 5 & 40 & 20 & 30 & & \\
\hline Pendiente (\%) & 20 & 2 & 5 & 10 & 30 & 10 & 10 & 50 & 5 & 5 & 20 & 5 & & \\
\hline Fm. Geológica & $\mathrm{H}$. & M. & $\mathrm{H}$. & $\mathrm{H}$. & $\mathrm{H}$. & $\mathrm{H}$. & $\mathrm{H}$. & E.C. & $\mathrm{H}$. & $\mathrm{H}$. & $\mathrm{H}$. & $\mathrm{H}$. & & \\
\hline Relevamiento & 25 & 26 & 27 & 28 & 29 & 30 & 31 & 32 & 33 & 34 & 35 & 36 & & \\
\hline P. philipianum & & & & 3 & & & & + & & & & & I & 0,26 \\
\hline T. usillo & & & & & & & & & & 3 & + & & I & 0,26 \\
\hline M. parviflora & & & & & & 3 & & & & & & & 1 & 0,25 \\
\hline A. lampa & & 3 & & & & & & & & & & & 1 & 0,25 \\
\hline B. aristidoides & + & + & + & + & + & & + & + & + & + & + & + & V & 0,09 \\
\hline A. incarnata & + & + & & + & & + & + & & & + & + & & III & 0,06 \\
\hline Li. cuneifolia & & + & & & + & & + & & & & + & & II & 0,03 \\
\hline E. serpens & + & & & + & & + & + & & & & & & II & 0,03 \\
\hline A. adscencionis & + & & & & & + & + & & & & & + & II & 0,03 \\
\hline T. strigosus & + & & + & & + & & & & & & & & II & 0,03 \\
\hline P. sanjuanensis & + & + & & & & + & & & & & & & II & 0,03 \\
\hline H. curassavicum & & + & & & & & + & & & + & & & ॥ & 0,03 \\
\hline L. tenuispinosum & & + & & & & & & & & + & & & 1 & 0,02 \\
\hline A. odorata & & & & & & + & & & & & + & & 1 & 0,02 \\
\hline Lepidium $s p$ & + & & & & & & + & & & & & & 1 & 0,02 \\
\hline H. ameghinoi & & & & & & & + & & + & & & & 1 & 0,02 \\
\hline
\end{tabular}

aroma, Aphyllocladus ephedroides, Senna aphylla, Psila retamoides y Bredemeyera colletioides.

Las cactáceas Tephrocactus aoracanthus, Opuntia sulphurea, Echinopsis leucantha y en menor medida Trichocereus strigosus y Pyrrhocactus sanjuanensis, están restringidas a los interfluvios, acompañando a Larrea divaricata. Se observó a T. aoracanthus formando grandes colonias. El estrato herbáceo posee una importante riqueza, pero con escasa cobertura y se ubica preferentemente en los bordes de cauces. Está constituido principalmente por Hyalis argentea, Pappophorum caespitosum, Aristida mendocina y Bouteloua aristidoides.

Unidad 4. Matorral bajo de Atriplex spegazzinii y Zuccagnia punctata

(Tabla 5; relevamientos: 37-43)
La fisonomía corresponde a lomadas altas y onduladas de material disgregado (huayquerías). El matorral está restringido a los sitios bajos entre las lomadas, con una escasa cobertura vegetal 15,0 $\%( \pm 8,6 \mathrm{SD})$ sobre los miembros neógenos $\mathrm{La}$ Colmena y Albardón (Fm. Loma de Las Tapias), con una superficie de $14,3 \mathrm{~km}^{2}(28,8 \%)$. La altitud promedio $(817,7 \mathrm{msnm} \pm 15,1 \mathrm{SD})$ es similar a la unidad 2.

El suelo es esquelético y muy erodable, con alta proporción de arcillas y limos con elevada salinidad en superficie, y en relación menor areniscas. En las lomadas con mayor pendiente se observan individuos esparcidos de Halophytum ameghinoi, mientras en los sitios bajos dominan arbustos de Atriplex spegazzinii, Zuccagnia punctata y Bulnesia retama, acompañados Prosopis flexuosa, 
Bol. Soc. Argent. Bot. 53 (3) 2018

Tabla 5. Matorral de Atriplex spegazzinii y Zuccagnia punctata. Valores de cobertura (\%). Otras especies halladas en un solo relevamiento con cobertura inferior al $1 \%$ : H. curassvicum (Relev. 37 ); $M$. ephedroides, P. philipianum, C. genistoides, N. noctiflora, T. dodoneifolia, Washingtonia sp. (Relev. 39); A. lampa (Relev. 40); O. sulphurea, M. albescens (Relev. 42); A. incarnata (Relev. 43). Fm. Geológica: Albardón (Alb.); Holoceno (H.).

\begin{tabular}{|c|c|c|c|c|c|c|c|c|c|}
\hline$x$ & $\begin{array}{l}\hat{i} \\
\stackrel{i}{N} \\
\infty \\
\% \\
o \\
o \\
0\end{array}$ & $\begin{array}{l}\overline{0} \\
\stackrel{0}{0} \\
\% \\
0 \\
0\end{array}$ & 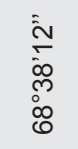 & 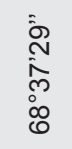 & $\begin{array}{l}\bar{n} \\
\bar{n} \\
\hat{n} \\
\infty \\
\infty \\
0\end{array}$ & 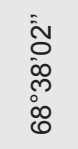 & $\begin{array}{l}0 \\
\text { it } \\
00 \\
\infty \\
\infty \\
0 \\
0\end{array}$ & \multirow{7}{*}{$\begin{array}{l}\frac{\pi}{0} \\
\frac{\pi}{\Phi} \\
0 \\
\Phi \\
0\end{array}$} & \multirow{7}{*}{ 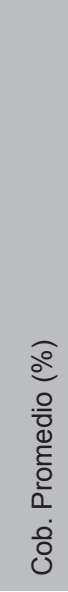 } \\
\hline Y & 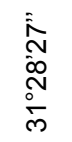 & 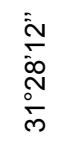 & 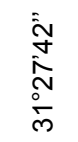 & 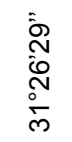 & 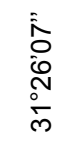 & 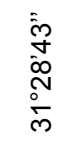 & 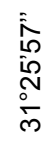 & & \\
\hline Altitud (msnm) & $\frac{\circ}{\infty}$ & $\underset{\infty}{\stackrel{\infty}{n}}$ & $\underset{\infty}{\infty}$ & $\frac{\widehat{N}}{\infty}$ & $\underset{\infty}{\mathbb{N}}$ & $\bar{\infty}$ & $\begin{array}{l}\infty \\
\infty \\
\sim\end{array}$ & & \\
\hline Cobertura (\%) & 10 & 10 & 20 & 10 & 5 & 20 & 10 & & \\
\hline Pendiente (\%) & 5 & 2 & 5 & 10 & 5 & 10 & 20 & & \\
\hline Fm. Geológica & Alb. & Alb. & Alb. & Alb. & Alb. & Alb. & $\mathrm{H}$. & & \\
\hline Relevamiento & 37 & 38 & 39 & 40 & 41 & 42 & 43 & & \\
\hline A. spegazzinii & 3 & + & 3 & + & + & 8 & 3 & V & 2,47 \\
\hline Z. punctata & + & 3 & 3 & + & & 8 & 3 & v & 2,46 \\
\hline B. retama & 3 & 3 & + & 3 & 3 & & & IV & 1,73 \\
\hline P. flexuosa & & 3 & 3 & 3 & & & 3 & III & 1,71 \\
\hline P. tetracantha & & + & & 3 & & & 3 & III & 0,87 \\
\hline A. aroma & & & 3 & & & 3 & + & III & 0,87 \\
\hline S. aphylla & & 3 & 3 & & & & + & III & 0,87 \\
\hline T. usillo & & 3 & & & & 3 & + & III & 0,87 \\
\hline C. praecox & & & 3 & & & 3 & & II & 0,86 \\
\hline T. aoracanthus & 3 & + & + & + & + & + & + & v & 0,51 \\
\hline La. cuneifolia & + & & 3 & & & & & II & 0,44 \\
\hline H. argentea & & & 3 & & & + & & II & 0,44 \\
\hline L. tenuispinosum & & & 3 & & & & & I & 0,43 \\
\hline P. chilensis & & & 3 & & & & & 1 & 0,43 \\
\hline P. retamoides & & & 3 & & & & & I & 0,43 \\
\hline B. salicifolia & & & 3 & & & & & 1 & 0,43 \\
\hline H. ameghinoi & & + & + & + & & & & III & 0,04 \\
\hline Li. cuneifolia & & + & + & & & + & & III & 0,04 \\
\hline T. crinita & & + & & + & & & + & III & 0,04 \\
\hline E. leucantha & + & & + & & & & & II & 0,03 \\
\hline T. strigosus & & + & & & & + & & II & 0,03 \\
\hline P. caespitosum & + & & + & & & & & II & 0,03 \\
\hline A. adscencionis & & & + & & + & & & II & 0,03 \\
\hline A. mendocina & & & + & & & + & & II & 0,03 \\
\hline S. kurtzii & & + & & + & & & & II & 0,03 \\
\hline T. ramossisima & & & + & + & & & & ॥ & 0,03 \\
\hline
\end{tabular}




\section{G. Almirón - Vegetación del Área Protegida "Loma de Las Tapias", San Juan}

Plectrocarpa tetracantha y Tricomaria usillo, entre otras. En el estrato inferior se observan pequeñas agrupaciones de Tephrocactus aoracanthus, Halophytum ameghinoi y Trichloris crinita, como especies más conspicuas.

\section{Discusión y ConClusiones}

El régimen pluviométrico estival con escasas lluvias torrenciales sumado a la fuerte insolación, elevadas temperaturas y evapotranspiración(Poblete \& Minetti, 1999), determinan fisonómicamente al área como un matorral abierto xerófilo de zigofiláceas y leguminosas acompañadas por otros arbustos, cactáceas y escasas herbáceas, típicos de la Provincia Fitogeográfica del Monte (Morello, 1958). A los factores de aridez se suma el efecto de la elevada proporción de arcillas y limos típicos de los sedimentos neógenos (huayquerías) carentes de materia orgánica y con tenores salinos elevados que restringen el desarrollo de la vegetación.

El análisis de componentes principales demuestra que la cobertura de la vegetación, la altitud, la pendiente y el tipo de afloramiento son determinantes de las comunidades, como se observa en otros estudios de vegetación realizados en ambientes desérticos (Márquez \& Dalmasso, 2003; Márquez et al., 2005; Martínez Carretero et al., 2005; Márquez et al., 2011).

Respecto a las otras áreas protegidas de San Juan en las que se han desarrollado estudios florísticos, Loma de Las Tapias posee algunas comunidades similares a las encontradas en La Ciénaga y Pedernal. En la Ciénaga, Márquez et al. (2011) establecen la "comunidad del Jarillal" ubicada en bajadas pedemontanas y laderas de serranías con Zuccagnia punctata y Larrea cuneifolia como especies dominantes y la "comunidad del chaguaral" también observada sobre laderas escarpadas y afloramientos rocosos, dominada por cojines de bromeliáceas (Deuterocohnia longipetala y Dickya velascana). Sin embargo, ambas comunidades poseen menor riqueza en Loma de las Tapias, debido probablemente a la mayor precipitación anual en La Ciénaga (100-200 mm).

En la Pampa del Acequión (Pedernal), tres comunidades con ciertas similitudes fueron observadas por Dalmasso \& Marquez (2004): comunidad de L. cuneifolia en valles, depresiones e interfluvios pedemontanos; comunidad de
L. divaricata en abanicos aluviales, conos de deyección y cauces temporarios y la comunidad de vegetación saxícola, donde domina $D$. longipetala. La mayor riqueza y cobertura general en Pedernal, posiblemente se deba a que este sitio se ubica a $50 \mathrm{~km}$ al suroeste de Loma de Las Tapias, a mayor altitud (de 850 a $2500 \mathrm{msnm}$ ), con más precipitaciones (que varían de este a oeste entre 186,5 y $269,4 \mathrm{~mm}$ ) y con temperatura media anual menor $\left(18^{\circ} \mathrm{C}\right)$.

A escala regional, el sistema de áreas protegidas del noroeste argentino (Jujuy, Catamarca, Salta, Tucumán, La Rioja y San Juan) es ineficiente en relación con la conservación de plantas endémicas según Godoy-Bürki et al. (2014). Estos autores proponen nuevos sitios específicos a proteger (en San Juan, la cordillera de Colangüil al oeste y el área central de la provincia). Sin embargo, en ese trabajo no se consideraron áreas protegidas ya existentes. En el centro de San Juan y coincidiendo con la posición geográfica de Loma de Las Tapias, destacan la importancia de priorizar la conservación de tres especies endémicas, encontradas en el presente estudio:

Pyrrhocactus sanjuanensis, una cactácea endémica de San Juan (Kiesling, 2003), restringida a los alrededores de la Sierra de Villicum $(3 \mathrm{~km}$ al noreste de Loma de Las Tapias), fue observada en las unidades 1, 2 y 3 con numerosos individuos.

Gomphrena cladotrichoides, también endémica de la provincia, que se distribuye en valles precordilleranos y en la región semiárida (Pedersen, 1994), fue encontrada al noreste del área (unidad 3) donde también se observaron dos especies endémicas interesantes: Aphyllocladus ephedroides cuya distribución alcanza las provincias de Catamarca, La Rioja y San Juan (Freire, 1996) y Prosopis argentina, arbusto psamófilo que se distribuye también en Catamarca, La Rioja, San Juan y Mendoza (Gomez Sosa \& Palacios; en Kiesling, 1994) .

En ciertos cauces temporarios (unidades 3 y 4) se observaron arbustos de Tamarix ramossisima, especie invasora de diversos ambientes de Argentina y otros países. Si bien la extrema aridez del sitio hace pensar que es difícil una propagación masiva, es sugerible su erradicación o al menos su control para evitar potenciales expansiones como ha ocurrido en otras áreas del planeta (Milton \& Dean, 2010). 
El impacto generado por los vehículos a campo traviesa (endurismo, cuatriciclos, ciclismo de montaña) deja un pasivo ambiental severo al erosionar el ya frágil suelo, afectando a diferentes escalas tanto a la vegetación (Brooks \& Lair, 2005; Navas Romero et al., 2016) como a la fauna. Durante las recorridas efectuadas, se constató un sinnúmero de huellas profundas antiguas y recientes que seccionan el paisaje, disminuyendo la calidad escénica. La presencia de un basurero con indicios de incineración en el noreste del área resulta un peligro potencial para la calidad ambiental del área.

Las cuatro unidades poseen situaciones complejas al considerar pautas de uso. A partir de las especies encontradas, es destacable la protección de la unidad 1 por la importante población de Pyrrhocactus sanjuanensis. También la unidad 3 resulta interesante, por la presencia de G. cladotrichoides y A. ephedroides y P. argentina.

Las otras dos unidades (2 y 4), poseen un factor agravante: la alta erodabilidad del suelo. En estas zonas es donde los vehículos a campo traviesa provocan cárcavas y la disgregación del frágil suelo, eliminando también la cubierta vegetal, ocasionando una pérdida de bienes y servicios ecosistémicos (Navas Romero et al., 2016) irrecuperable. Es propicio que en estas áreas se proteja la vegetación arbustiva que atenúa el proceso erosivo natural y antrópico. Posteriores estudios de fauna serán también necesarios para tener una visión mas representativa del ambiente natural del área protegida.

\section{Agradecimientos}

A Márquez, J.; Martínez Carretero, E. y Contreras, V. por sus valiosas sugerencias y al personal de la Sec. de Estado de Ambiente y Desarrollo Sustentable de la provincia.

\section{Bibliografía}

APODACA, M., J. CRISCI \& L. KATINAS. 2015. Las provincias fitogeográficas de la República Argentina: definición y sus principales áreas protegidas. En: El deterioro de los suelos y del ambiente en la Argentina, pp. 79-101. Ed. Fundación para la Educación, la Ciencia y la Cultura, Buenos Aires.
BRAUN-BLANQUET, J. 1979. Fitosociología. Bases para el estudio de las comunidades vegetales. Ed. H. Blume, Madrid.

BROOKS, M. \& B. LAIR. 2005. Ecological effects of vehicular routes in a desert ecosystem. Report prepared for the United States Geological Survey, Recoverability and Vulnerability of Desert Ecosystems Program.

CABRERA, A. 1976. Regiones fitogeográficas argentinas. En: Enciclopedia argentina de agricultura y jardinería. Tomo 2. 2da edición. pp. 1-85. Ed. Kugler, W. Buenos Aires.

CONTRERAS, V. 1981. Características bioestratigráficas del Terciario de Lomas de Las Tapias, departamento Ullum, provincia de San Juan. Actas $8^{\text {vo }}$ Congreso Geológico Argentino. Revista de la Asociación Geológica Argentina 4: 813-822.

CONTRERAS, V. \& J. BARALDO. 2011. Calibration of the Chasicoan-Huayquerian stages boundary (Neogene), San Juan, western Argentina. En: Cenozoic Geology of the Central Andes of Argentina. Pp. 111-121. Ed. SCS Publisher. Salta.

DI RIENZO, J., F. CASANOVES, M. BALZARINI, L. GONZALEZ, M. TABLADA \& C. ROBLEDO. 2016. InfoStat. Grupo InfoStat, FCA-UNC, Argentina.

GODOY-BÜRKI, A., P. ORTEGA-BAES, J. SAJAMA \& L. AAGESEN. 2014. Conservation priorities in the Southern Central Andes: mismatch between endemism and diversity hotspots in the regional flora. Biodivers Conserv. 23: 81-107.

KÖPPEN W. 1931. Grundriss der Klimakunde. Ed. De Gruite, Berlin.

LIOTTA, M. 2011. Los suelos en el Valle del Tulum, Ullum-Zonda. Informe Técnico INTA. Estación Experimental Agropecuaria San Juan.

MÁRQUEZ, J. \& A. DALMASSO. 2003. Las comunidades vegetales de los ambientes húmedos del Parque Nacional El Leoncito, San Juan, Argentina. Multequina 12: 55-67.

MÁRQUEZ, J., E. MARTÍNEZ CARRETERO, A. DALMASSO, G. PASTRÁN \& G. ORTIZ. 2005. Las Áreas Protegidas de la Provincia de San Juan (Argentina) II. La Vegetación Del Parque Provincial Ischigualasto. Multequina 14: 1-27.

MÁRQUEZ, J., Y. RIPOLL, M. ARIZA, E. MARTÍNEZ CARRETERO, \& A. DALMASSO. 2011. Naturaleza y cultura de La Ciénaga. Ed. UNSJ. San Juan.

MÁRQUEZ, J., E. MARTÍNEZ CARRETERO, \& A. DALMASSO. 2017. Provincias Fitogeográficas de la Provincia de San Juan. En: Martínez Carretero, E. y A. García (eds.), San Juan Ambiental, pp: 187-197. Mendoza. 


\section{G. Almirón - Vegetación del Área Protegida "Loma de Las Tapias”, San Juan}

MÁRQUEZ, J. \& G. PASTRÁN. 2017. Las Áreas Naturales Protegidas de la Provincia de San Juan. En: Martínez Carretero, E. y A. García (eds.). San Juan Ambiental, pp: 459-478. Mendoza.

MARTÍNEZ CARRETERO, E., A. DALMASSO, J. MÁRQUEZ \& G. PASTRÁN. 2008. Comunidades Vegetales y Unidades Fitogeográficas. En: UNSJ. San Juan (Ed.), Diversidad biológica y cultural de los altos andes centrales de Argentina. Linea de base de la reserva de biosfera San Guillermo-San Juan. pp. 113-152.

MILANA, J. 1991. Sedimentología y magnetoestratigrafía de formaciones cenozoicas en el área de Mogna y su inserción en el marco tectosedimentario de la Precordillera Oriental (Tesis doctoral, Univ. Nac. de San Juan).

MILTON, S. \& R. DEAN. 2010. Plant invasions in arid areas: special problems and solutions: a South African perspective. Biol. Invasions. 12: 3935-3948.

NAVAS ROMERO, A., M. HERRERA-MORATTA, A. DALMASSO \& R. MAURICIO. 2016. Efecto del motocross en la vegetación y en las propiedades fisicoquímicas del suelo en la zona aledaña a la Reserva Divisadero Largo, Mendoza. Actas de la II Reunión Argentina de Jóvenes Botánicos. Soc. Argentina de Botánica.
POBLETE, A. \& J. MINETTI. 1999. Configuración espacial del clima de San Juan. Síntesis del Cuaternario de la Provincia de San Juan. $11^{\circ}$ Reunión de Campo del Cuaternario. INGEO, Universidad Nacional de San Juan, San Juan.

RODRÍGUEZ, D. 2004. Estudio sedimentológico y estratigráfico del Neógeno superior de Loma de Las Tapias, con el fin de interpretar la evolución del antiguo rio San Juan (Tesis de licenciatura, Univ. Nac. de San Juan).

SUVIRES, G. \& V. CONTRERAS. 2011. Late Cenozoic geology and geomorphology of the Loma de Las Tapias area, San Juan, west central Argentina. En: Cenozoic Geology of the Central Andes of Argentina, pp. 427-436. Ed. SCS Publisher. Salta, Argentina.

ZULOAGA, F. O., MORRONE, O., \& M. J. BELGRANO. 2008. Catálogo de Las Plantas Vasculares Del Cono Sur: (Argentina, Sur de Brasil, Chile, Paraguay y Uruguay). Monogr. Syst. Bot. Missouri Bot. Gard. 107: 1-3348.

Recibido el 14 de diciembre de 2017, aceptado el 5 de septiembre de 2018. Editor: Guillermo Funes. 
Bol. Soc. Argent. Bot. 53 (3) 2018

Apéndice 1. Listado de plantas vasculares encontradas en el área protegida.

\begin{tabular}{|c|c|c|c|}
\hline Familia & Especie & Familia & Especie \\
\hline Amaranthaceae & Gomphrena cladotrichoides Suess. & Halophytaceae & $\begin{array}{l}\text { Halophytum ameghinoi } \\
\text { (Speg.) Speg. }\end{array}$ \\
\hline Amaryllidaceae & Zephyranthes filifolia Herb. ex Kraenzl. & \multirow{2}{*}{ Loasaceae } & $\begin{array}{l}\text { Mentzelia albescens (Gillies } \\
\text { ex Arn.) Griseb. }\end{array}$ \\
\hline Apocynaceae & $\begin{array}{l}\text { Araujia odorata (Hook. \& } \\
\text { Arn.) Fontella \& Goyder }\end{array}$ & & $\begin{array}{l}\text { Mentzelia parvifolia Urb. } \\
\text { \& Gilg ex Kurtz }\end{array}$ \\
\hline Araceae & Washingtonia sp. & Loranthaceae & $\begin{array}{l}\text { Ligaria cuneifolia (Ruiz } \\
\text { \& Pav.) Tiegh. }\end{array}$ \\
\hline \multirow{2}{*}{ Boraginaceae } & Heliotropium curassavicum L. & Malpighiaceae & Tricomaria usillo Hook. \& Arn. \\
\hline & Phacelia aff. sanzinii Hicken & Malvaceae & Gaya parviflora (Phil.) Krapov. \\
\hline Brassicaceae & Lepidium aff. spicatum Desv. & \multirow{3}{*}{ Nictaginaceae } & $\begin{array}{l}\text { Mirabilis ovata (Ruiz \& } \\
\text { Pav.) F. Meigen }\end{array}$ \\
\hline Bromeliaceae & Deuterocohnia longipetala (Baker) Mez & & Bougainvillea spinosa (Cav.) Heimerl \\
\hline \multirow{4}{*}{ Cactaceae } & $\begin{array}{l}\text { Echinopsis leucantha (Gillies } \\
\text { ex Salm-Dyck) Walp. }\end{array}$ & & Allionia incarnata L. \\
\hline & $\begin{array}{l}\text { Opuntia sulphurea Gillies ex } \\
\text { Salm-Dyck var. sulphurea }\end{array}$ & Olacaceae & $\begin{array}{l}\text { Ximenia americana L. var. } \\
\text { argentiniensis De Filipps }\end{array}$ \\
\hline & $\begin{array}{l}\text { Pyrrhocactus sanjuanensis (Speg.) Backeb. } \\
\text { Tephrocactus aoracanthus (Lem.) Lem. } \\
\text { Trichocereus candicans (Gillies } \\
\text { ex Salm-Dyck) Britton \& Rose }\end{array}$ & \multirow{7}{*}{ Poaceae } & $\begin{array}{l}\text { Aristida adscensionis L. } \\
\text { Aristida mendocina Phil. } \\
\text { Bouteloua aristidoides } \\
\text { (Kunth) Griseb. }\end{array}$ \\
\hline & $\begin{array}{l}\text { Trichocereus strigosus (Salm- } \\
\text { Dyck) Britton \& Rose }\end{array}$ & & Cottea pappophoroides Kunth \\
\hline Capparaceae & Capparis atamisquea Kuntze & & Pappophorum caespitosum R.E. Fr. \\
\hline & Atriplex argentina Speg. & & Pappophorum phillippianum Parodi \\
\hline Chenopodiaceae & Atriplex lithophila Soriano ex Mulgura & & $\begin{array}{l}\text { Sporobolus pyramidatus } \\
\text { (Lam.) Hitchc. }\end{array}$ \\
\hline & Atriplex lampa (Moq.) D. Dietr. & & Jarava ichu Ruiz \& Pav. var. ichu \\
\hline \multirow{9}{*}{ Compositae } & Aphyllocladus ephedroides Cabrera & & Trichloris crinita (Lag.) Parodi \\
\hline & Baccharis salicifolia (Ruiz \& Pav.) Pers. & Polygalaceae & $\begin{array}{l}\text { Bredemeyera colletioides } \\
\text { (Phil.) Chodat }\end{array}$ \\
\hline & Cyclolepis genistoides Gillies ex D. Don & & Grahamia bracteata Hook. \& Arn. \\
\hline & $\begin{array}{l}\text { Hyalis argentea D. Don ex } \\
\text { Hook. \& Arn. var. argentea }\end{array}$ & Portulacaceae & Portulaca grandiflora Hook. \\
\hline & Porophyllum obscurum (Spreng.) DC. & \multirow{6}{*}{ Solanaceae } & Lycium chilense Miers \\
\hline & $\begin{array}{l}\text { Baccharis spartioides (Hook. } \\
\text { \& Arn. ex DC.) J. Remy }\end{array}$ & & $\begin{array}{l}\text { Lycium tenuispinosum Miers } \\
\text { var. tenuispinosum }\end{array}$ \\
\hline & Senecio aff. pinnatus Poir. var. pinnatus & & Lycium boerhaviaefolium L. f. \\
\hline & $\begin{array}{l}\text { Tessaria dodoneifolia (Hook. } \\
\text { \& Arn.) Cabrera }\end{array}$ & & Nicotiana noctiflora Hook. \\
\hline & Thymophylla pentachaeta (DC.) Small & & Sclerophylax aff. kurtzii Di Fulvio \\
\hline Euphorbiaceae & Euphorbia serpens Kunth var. serpens & & Solanum elaeagnifolium Cav. \\
\hline
\end{tabular}


M. G. Almirón - Vegetación del Área Protegida "Loma de Las Tapias", San Juan

\begin{tabular}{|c|c|c|c|}
\hline Familia & Especie & Familia & Especie \\
\hline \multirow{10}{*}{ Fabaceae } & Acacia aroma Gillies ex Hook. \& Arn. & Tamaricaceae & Tamarix ramosissima Ledeb. \\
\hline & Cercidium praecox (Ruiz \& & \multirow{4}{*}{ Verbenaceae } & Aloysia castellanosii Moldenke \\
\hline & Mimosa ephedroides (Gillies & & Aloysia aratissima (Gillies \& \\
\hline & ex Hook. \& Arn.) Benth. & & Hook. ex Hook.) Tronc. \\
\hline & Prosopis argentina Burkart & & Glandularia perakii \\
\hline & Prosopis chilensis (Molina) & \multirow{5}{*}{ Zigophyllaceae } & Bulnesia retama (Gillies ex \\
\hline & Stuntz emend. Burkart & & Hook. \& Arn.) Griseb. \\
\hline & Prosopis flexuosa DC. var. & & Larrea cuneifolia Cav. \\
\hline & Senna anhylla (Cav) H S Irwin \& Barneby & & Larrea divaricata Cav \\
\hline & Zuccagnia punctata Cav. & & $\begin{array}{l}\text { Plectrocarpa tetracantha } \\
\text { Gillies ex Hook. \& Arn. }\end{array}$ \\
\hline
\end{tabular}


